AperTO - Archivio Istituzionale Open Access dell'Università di Torino

\title{
Addition of docetaxel to androgen deprivation therapy for patients with hormone-sensitive metastatic prostate cancer: A systematic review and meta-analysis
}

\section{This is the author's manuscript}

Original Citation:

Availability:

This version is available http://hdl.handle.net/2318/1568961

since 2016-10-17T14:16:43Z

Published version:

DOI:10.1016/j.eururo.2015.09.013

Terms of use:

Open Access

Anyone can freely access the full text of works made available as "Open Access". Works made available under a Creative Commons license can be used according to the terms and conditions of said license. Use of all other works requires consent of the right holder (author or publisher) if not exempted from copyright protection by the applicable law. 
1 Addition of docetaxel to androgen deprivation therapy for patients with hormonesensitive, metastatic prostate cancer: a systematic review and meta-analysis.

3

\section{(8)}

Marcello Tucci $^{1}$, Valentina Bertaglia ${ }^{1}$, Francesca Vignani ${ }^{1}$, Consuelo Buttigliero ${ }^{1}$, Cristian

Fiori² $^{2}$ Francesco Porpiglia ${ }^{2}$, Giorgio Vittorio Scagliotti ${ }^{1}$ and Massimo Di Maio ${ }^{1}$

${ }^{1}$ Division of Medical Oncology, Department of Oncology, University of Turin, San Luigi

Gonzaga Hospital, Orbassano, Turin, Italy.

2Division of Urology, Department of Oncology, University of Turin, San Luigi Gonzaga

Hospital, Orbassano, Turin, Italy.

Word count:

Abstract: 300 words

Text (including abstract): 4070 words

Keywords: Docetaxel; Systematic review; Meta-analysis; Androgen-deprivation treatment; Hormone-sensitive prostate cancer; Chemotherapy.

\section{Corresponding author:}

Prof. Massimo Di Maio

Department of Oncology

University of Turin

San Luigi Gonzaga Hospital

Regione Gonzole 10, 10043 Orbassano (TO), Italy

Phone: +39 0119026017

Fax: +390119015184

Email: massimo.dimaio@unito.it 
Abstract

Context: Several randomized clinical trials (RCTs) have recently tested the early addition of docetaxel to androgen deprivation therapy (ADT) in hormone-sensitive, metastatic prostate cancer.

Objective: To perform a systematic review and meta-analysis of RCTs evaluating the combination of docetaxel and ADT in hormone-sensitive, metastatic prostate cancer. Primary endpoint was overall survival (OS). Secondary endpoint was progression-free survival. Exploratory subgroup analysis according to high-volume vs. low-volume disease was performed.

Evidence acquisition: A systematic review of PubMed/Medline, Embase, and proceedings of main International meetings was performed in June 2015 and updated in August 2015. Three trials were selected for inclusion.

Evidence synthesis: Overall, 2951 patients were included in the 3 trials. Two trials enrolled only metastatic patients, while in the third trial $61 \%$ were metastatic: overall, metastatic patients were 2262 (951 docetaxel+ADT, 1311 ADT alone). Most patients had a good performance status. In metastatic patients, the addition of docetaxel was associated with improved OS (hazard ratio $[\mathrm{HR}] 0.73,95 \% \mathrm{Cl} 0.60-0.90, \mathrm{p}=0.002$ ), with non significant heterogeneity among the 3 trials. Considering the whole study population (2951 patients), addition of docetaxel was associated with a similar OS improvement $(\mathrm{HR} 0.74,95 \% \mathrm{Cl}$ 0.61-0.91, $\mathrm{p}=0.003)$. Although with limited statistical power, no significant interaction was demonstrated between the addition of docetaxel and the high or low volume of disease $(p=0.5)$. The addition of docetaxel was associated with improvement in progression-free survival (metastatic patients: HR 0.63, 95\%Cl 0.57-0.70, p<0.001). 
55 Conclusions: This meta-analysis shows a significant OS benefit from concomitant

56 administration of docetaxel and ADT in patients with metastatic, hormone-sensitive 57 prostate cancer.

58 Patient summary: We synthesized the evidence available about the early administration

59 of docetaxel in patients starting hormonal treatment for metastatic prostate cancer. Based

60 on results of this meta-analysis, we believe that the combination of chemotherapy and

61 hormonal treatment should be considered in fit patients.

62 
Introduction

Prostate cancer is the second most frequently diagnosed cancer in men and is the second leading cause of cancer death in male patients in United States and Europe [1]. Although localized prostate cancer may be successfully treated with radical prostatectomy and external beam radiation, many patients will subsequently develop metastatic disease [2]. In addition, in the United States, the proportion of patients presenting with advanced stage at first diagnosis of prostate cancer is $4-5 \%$ for distant disease and $10-12 \%$ for regional disease [3]. Androgen deprivation therapy (ADT) by medical or surgical castration is the mainstay of treatment for locally advanced and metastatic prostate cancer, because androgen receptor (AR) pathway plays a key role in the development and progression of prostate cancer cells [4]. Although ADT is able to induce biochemical and clinical response in more than $90 \%$ of patients, after a median of $24-36$ months patients experience phase III trials have demonstrated the efficacy of two new-generation hormonal therapies

progression to castration-resistant prostate cancer (CRPC), despite persisting low testosterone levels [5].

Until very recently, chemotherapy with docetaxel has been the only effective treatment for CRPC patients. In detail, the randomized clinical trial (RCT) TAX327 demonstrated that docetaxel plus prednisone prolonged overall survival (OS) compared to mitoxantrone plus prednisone [6]. Another RCT, the SWOG-9916 study, also demonstrated that the treatment with docetaxel, estramustine and dexamethasone increased median OS by two months compared to mitoxantrone and prednisone [7]. Based on these results, docetaxel was the first cytotoxic drug to demonstrate an improvement in OS in prostate cancer. More recently, several new agents, able to modify the natural history of disease, have been introduced in clinical practice. Results from (abiraterone [8] and enzalutamide [9]), an immunotherapy (sipuleucel-T [10]), a new 
89 microtubule-targeting chemotherapy (cabazitaxel [11]) and an alpha-emitter (radium 223

90 [12]), all able to prolong OS.

Nowadays, progression of CRPC is known to be due to the onset of a number of resistance mechanism induced by the selective pressure of endocrine therapy [13-18].

Castration is able to induce clonal selection and subsequent growth of androgenindependent cellular clones [19]. Hormone-sensitive prostate cancer should be considered a heterogeneous disease, characterized by the coexistence of both AR-positive and ARnegative tumor cells.

In this biological context, patients with hormone-sensitive prostate cancer may benefit of chemotherapy in association with endocrine therapy, targeting also AR-negative cells and delaying the development of resistance mechanisms. In the "pre-docetaxel" era, several RCTs investigated the combination of endocrine therapy with other cytotoxic drugs in hormone-sensitive prostate cancer patients, but none of these studies showed a significant and convincing advantage $[20,21]$. In the last two years, the results of three different clinical trials (GETUG-AFU 15 [22], CHAARTED - E3805 [23] and STAMPEDE [24]), that investigated the combination of docetaxel and ADT in hormone sensitive disease, have been made available to scientific community. The aim of this systematic review is to conduct a meta-analysis of RCTs that evaluated the combination of docetaxel with ADT vs. ADT alone, in hormone-sensitive metastatic prostate cancer, in order to assess the impact of this therapeutic option in terms 109 of overall survival. 


\section{Evidence acquisition}

\section{Identification of eligible trials}

Full protocol of the review is available on request from the corresponding author.

114 Search was performed in June 2015 and updated in August 2015, to identify all

115 randomized trials testing the addition of docetaxel to ADT in patients with hormone-naive

116 metastatic prostate cancer. Literature search was performed using PubMed, EMBASE,

117 Medline, Cochrane Library. The following key-words were used: (prostate cancer) AND

118 docetaxel AND (random*). References of the selected articles were also checked to

119 identify further eligible trials. Furthermore, proceedings of the main International meetings

120 (American Society of Clinical Oncology [ASCO] annual meeting, ASCO Genitourinary

121 symposium, European Society of Medical Oncology, European Association of Urology),

122 were searched from 2010 onwards for relevant abstracts. Trials enrolling both patients with

123 metastatic disease and patients without metastases were eligible (details about subgroups

124 were collected as specified below). Trials enrolling only patients without metastases

$125[25,26]$ were excluded. When more than one report was available describing results of the

126 same trial, the most recent information (corresponding to a longer follow-up and a higher

127 number of events) was considered in the analysis.

129 Data collection and study quality

130 For each eligible trial, the following data were collected, if available:

131 - main inclusion criteria: age, performance status, stage, Gleason score, prostatespecific antigen (PSA) at randomization, presence of visceral metastases, volume(high vs. low) of metastatic disease, previous treatments;

- details of study treatment: type of ADT allowed, schedule and number of cycles of docetaxel planned in experimental arm, timing of docetaxel start compared to ADT 
initiation in experimental arm, number of docetaxel cycles actually administered (median, range), proportion of patients completing planned docetaxel cycles, proportion of patients needing dose reduction of docetaxel;

- study design: primary endpoint, study hypothesis;

- patients' enrolment and follow-up: date of start and date of end of accrual; number of patients assigned to experimental arm (docetaxel + ADT), number of patients assigned to control arm (ADT alone), median follow-up;

- Overall survival [OS]: number of deaths in each arm, median OS, hazard ratio with $95 \%$ confidence interval, $p$ value, details of subgroup analysis of metastatic patients (for trials enrolling both M0 and M1 patients), details of subgroup analysis in "highvolume" patients and "low-volume" patients;

- Progression-free survival [PFS]: number of events in each arm, median PFS, hazard ratio with $95 \%$ confidence interval, $p$ value, details of subgroup analysis of metastatic patients (for trials enrolling both $\mathrm{M0}$ and $\mathrm{M} 1$ patients).

For each study, the quality of the randomization process was evaluated based on the information available in the publication [22, 23] or in the study protocol [24].

\section{Statistical Methods}

After data were abstracted, analysis was performed the Review Manager (RevMan 5.3) software. In all the trials included, efficacy data were analysed from all randomly assigned patients on an intention-to-treat basis. Primary endpoint of the meta-analysis was overall survival. Secondary endpoint was biochemical progression-free survival (bPFS).

Definition of bPFS was different in the three trials and is reported in Supplemental table A1. 
For both overall survival and bPFS, summary measure was hazard ratio (with 95\%

confidence interval). A random-effects model was applied. Statistical heterogeneity between studies was examined using the $\chi^{2}$ test and the $\mathrm{I}^{2}$ statistic.

Main analysis was performed considering the 3 comparisons of docetaxel + ADT vs. ADT alone. In one trial [24], a further experimental arm was reported, testing the addition of docetaxel + zoledronic acid to ADT alone. Since the addition of zoledronic acid alone did not show any significant efficacy compared to ADT, we decided to perform an exploratory analysis adding also this comparison to the analysis of docetaxel. However, since that trial used the same control arm for the two comparisons (docetaxel + ADT vs. ADT alone, and docetaxel + zoledronic acid + ADT vs. ADT alone), the weight of each comparison was reduced according to a correction factor equal to the number of events actually observed in the trial, divided by the number of events taken into account in the analysis (where the control arm was counted twice). This correction resulted in a prudential increase in the width of the confidence interval for the estimated hazard ratio of each comparison.

For overall survival, the subgroup analysis of patients according to disease volume ("high-volume" vs "low-volume") was available for two of the three trials $[27,23]$. In both trials, "high-volume" disease was defined as the presence of at least 4 bone lesions and at least 1 lesion in any bone beyond the spine / pelvis, or the presence of visceral metastasis. Patients without these conditions were classified as "low-volume". No subgroup analysis of progression-free survival according to disease volume was available.

\section{Role of funding source}

There was no funding source for this review. All authors had full access to all the data and the corresponding author (MDM) had final responsibility for the decision to submit for publication. 


\section{Evidence synthesis}

\section{Characteristics and quality of the trials}

The selection process of trials eligible for the meta-analysis is reported in Supplemental

Figure 1. In the search updated in August 2015, out of the 466 papers published in extenso, 464 were excluded, while two (GETUG-AFU 15 and CHAARTED - E3805) were found eligible for inclusion $[22,23]$. One further eligible trial (STAMPEDE) was found searching the proceedings of the main International meetings [24]. Furthermore, an updated report of the already published GETUG-AFU 15 trial, with longer follow-up and a higher number of events for analysis, was available [27].

Main characteristics of the three available trials are described in Table 1. In all the trials, patients assigned to experimental arm received docetaxel $75 \mathrm{mg} / \mathrm{m}^{2}$, for a maximum of $6[23,24]$ or 9 cycles [22]. The maximum interval since ADT start allowed to start docetaxel ranged from 2 to 4 months: in the GETUG-AFU 15 trial about half of the patients had started ADT within 15 days of enrolment [22]; in the CHAARTED - E3805 trial, median time from ADT to randomization was slightly higher than 1 month in both arms [23].

According to description available in the publication for 2 trials $[22,23]$ and in the study protocol for the third trial [24], quality of randomization process was judged adequate in all the 3 trials.

\section{Patients' characteristics}

Overall, 2951 patients were included in the 3 trials included in the meta-analysis, $1181(40 \%)$ assigned to docetaxel + ADT, and 1770 (60\%) assigned to ADT alone (Table 
212 of the patients had a good performance status. Two of the trials $[22,23]$ enrolled only

213 metastatic patients, while in the STAMPEDE trial [24] metastatic patients were $61 \%$ of total

214 study population: overall, metastatic patients were 2262 (951 docetaxel+ADT, 1311 ADT

215 alone). Patients with metastatic disease at diagnosis were $71 \%$ in the GETUG-AFU 15 trial 216 and $73 \%$ in the CHAARTED - E3805 trial; 94\% of patients enrolled in the STAMPEDE trial 217 had not received previous local therapy. Patients with high-volume disease were $48 \%$ in 218 the GETUG-AFU 15 trial, and 65\% in the CHAARTED - E3805 trial; this information was 219 not available in the STAMPEDE trial.

\section{Treatment compliance and toxicity}

Median number of docetaxel cycles actually administered was 8 in the GETUG-AFU 15 trial [22], 6 in the CHAARTED - E3805 [23] and 6 in the STAMPEDE trial [24].

Proportion of patients completing the planned number of cycles was $48 \%$ in the GETUGAFU 15 trial (9 planned cycles), 86\% in the CHAARTED - E3805 trial (6 planned cycles) and $76 \%$ in the STAMPEDE trial (6 planned cycles). Proportion of patients needing dose reduction was 11\% in the GETUG-AFU 15 trial and 26\% in the CHAARTED -E3805 trial, while this information was not available in the report of the STAMPEDE trial. haematologic toxicity (anemia, thrombocytopenia, neutropenia), fatigue, gastro-intestinal toxicity (nausea, vomiting, constipation, diarrhea), alopecia, sensory neuropathy, stomatitis/mucositis, nail changes and peripheral edema. In all the 3 trials, the addition of 233 docetaxel was associated to higher incidence of febrile neutropenia: $8 \%, 6 \%$ and $12 \%$ in the GETUG-AFU 15, in the CHAARTED - E3805 and in the STAMPEDE trial, versus 0\%, not reported and 1\% with ADT alone in the 3 trials respectively. 
Number of events and OS data reported in each trial are summarized in Table 3.

Overall, 916 deaths were recorded for the main comparison (docetaxel + ADT vs. ADT alone) in metastatic patients. As shown in Figure 1 (panel A), the addition of docetaxel to ADT in metastatic patients was associated with a statistically significant benefit in overall survival (hazard ratio $[\mathrm{HR}] 0.73,95 \%$ confidence interval $[\mathrm{Cl}] 0.60-0.90, \mathrm{p}=0.002$ ).

There was no evidence of statistically significant heterogeneity among the three trials $(p=$ $\left.0.15, \mathrm{I}^{2}=48 \%\right)$. In the whole study population, including also the minority of non-metastatic patients (Figure 1, panel B), the addition of docetaxel to ADT was associated with a similar, statistically significant benefit in overall survival $(\mathrm{HR} 0.74,95 \% \mathrm{Cl} 0.61-0.91, \mathrm{p}=$ 0.003). Very similar results were obtained in the exploratory analysis including also the docetaxel + zoledronic acid arm of the STAMPEDE trial: HR $0.74(95 \% \mathrm{Cl} 0.63-0.88$, $\mathrm{p}<0.001)$ considering only metastatic patients (Figure 1, panel C), $\mathrm{HR} 0.76(95 \% \mathrm{Cl} 0.64-$ 0.89, $p=0.001$ ) in all patients (Figure 1, panel $\mathbf{D}$ ). "low-volume" disease enrolled in the GETUG-AFU 15 and in the CHAARTED - E3805 trial

(Figure 2). The test for difference of efficacy among the two subgroups did not

254 demonstrate a statistically significant interaction $(p=0.5)$. Hazard ratio for the addition of docetaxel to ADT was $0.67(95 \% \mathrm{Cl} 0.51-0.88)$ in patients with "high-volume" disease and $0.80(95 \% \mathrm{Cl} 0.49-1.32)$ in patients with "low-volume" disease.

\section{Progression-free survival}

As shown in Figure 3 (panel A), the addition of docetaxel to ADT in metastatic patients was associated with a statistically significant benefit in progression-free survival (HR $0.63,95 \% \mathrm{Cl} 0.57-0.70, \mathrm{p}<0.001)$, without significant heterogeneity among the three trials $\left(p=0.7, l^{2}=0 \%\right)$. The same benefit was shown considering the whole study population, including the minority of patients without metastates ( $\mathrm{HR} 0.63,95 \% \mathrm{Cl} 0.57-$ 
$2640.70, p<0.001)$ (Figure 3, panel B). Very similar results were obtained in the exploratory 265 analysis including also the docetaxel + zoledronic acid arm of the STAMPEDE trial: HR $2660.63(95 \% \mathrm{Cl} 0.56-0.70, \mathrm{p}<0.001)$ in metastatic patients (Figure 3, panel C), HR 0.63 $267(95 \% \mathrm{Cl} 0.57-0.70, \mathrm{p}<0.001)$ in all patients (Figure 3, panel D). 
Conclusions.

This meta-analysis shows that the addition of docetaxel to ADT in patients with metastatic, hormone-sensitive prostate cancer is associated with a significant improvement in overall survival and progression-free survival.

A quantitative synthesis of the evidence currently available about this treatment strategy can be really helpful for clinical decisions, because three recent, different phase III trials (GETUG-AFU-15 [22,27], CHAARTED - E3805 [23], STAMPEDE [24]) tested the activity of docetaxel in combination with endocrine therapy in the "early" setting of hormone-sensitive prostate cancer. To the best of our knowledge, there are no other trials conducted with docetaxel in the same setting, and this meta-analysis represents the synthesis of all the evidence produced to date. Notably, in GETUG-AFU-15 trial, the first trial to be published, the concomitant administration of docetaxel with ADT versus ADT alone did not show a significant impact in terms of OS $[22,27]$. On the contrary, CHAARTED - E3805 trial showed a significant OS improvement for ADT plus docetaxel [28], adding fuel to the scientific debate about the opportunity of this therapeutic option in hormone-sensitive prostate cancer patients. In our meta-analysis, that also included the recent results of the "third- comer", the STAMPEDE trial [24], the addition of docetaxel to ADT in metastatic patients was associated with a statistically significant increase in overall survival, with a moderate, non significant heterogeneity among the three available RCTs. Of note, the absence of statistical heterogeneity increases the validity of the result, allowing a global, unambiguous interpretation of all the evidence available. Of course, a meta-analysis based on individual patient data (IPD) would represent the best synthesis of evidence, allowing for data checking, updated follow-up compared to publications, calculation and comparison of times to events, and for investigation of treatment heterogeneity in subgroups [29]. However, in the absence of IPD meta-analysis, a meta- 
analysis based on abstracted data can be considered an acceptable surrogate, allowing a timely synthesis of all the available trials.

The efficacy showed by docetaxel in combination with ADT in hormone-sensitive patients is not surprising due to strong biological basis. Recent evidences show that one of the mechanisms responsible for progression from hormone-sensitive to castrationresistant phase of disease is the clonal selection and proliferation of pre-existing ARindependent cells, able to survive in a low androgen levels environment [19]. Therefore it is reasonable to assume that, since its onset, prostate cancer is a heterogeneous disease where coexist AR-positive and AR-negative cells $[19,30]$. Both these cellular clones are likely involved in progression to castration-resistant disease [19]. Docetaxel administration concurrent to ADT in hormone-sensitive prostate cancer patients allows to inhibit the growth of the pre-existing AR-insensitive clones, killing these cells earlier when they are still a small number and before the development of multiple escape mechanisms. Moreover, preclinical data show that the adaptive response to ADT by prostate cancer cells is mediated by both ligand-dependent AR activation and ligand-independent AR activation and by mechanisms of progression bypassing AR signaling $[19,31]$. Taxanes are able to interfere with several steps of these resistance mechanisms. Emerging preclinical data demonstrated that taxanes could inhibit AR signaling pathway [32]. In fact, these cytotoxic drugs interfere with polymerization of microtubules, blocking AR nuclear translocation and AR-induced gene expression [32,33]. Therefore docetaxel could act synergistically with endocrine therapy, because it impairs AR activity [32,33]. Additionally, chemotherapy may also kill cells that escape ADT through activation of AR-independent survival pathways [34].

From a clinical point of view, there are several potential advantages in administering chemotherapy to metastatic prostate cancer patients in an early phase of disease. In the hormone-sensitive setting, patients are, on average, in better clinical conditions compared 
320 to castration-resistant setting, due to lower burden of disease. Consequently, they are able

321 to better tolerate chemotherapy and to maintain adequate drug dose intensity. Moreover, a

322 greater number of patients is eligible for chemotherapy; in the castration-resistant setting,

323 on the contrary, a relevant number of patients cannot receive chemotherapy, due to

324 worsening of performance status and clinical conditions.

Our meta-analysis shows an improvement in OS that is not only statistically significant, but also clinically relevant. The addition of docetaxel to ADT is associated with a $27 \%$ reduction in the risk of death of metastatic patients (Hazard Ratio 0.73), and the reduction in the risk of death is as high as 33\% in patients with high-volume disease (Hazard Ratio 0.67). In absolute terms, such a benefit is rarely obtained in the setting of advanced solid tumors: difference in median OS for metastatic patients was more than 13 months in the CHAARTED - E3805 trial [23], and 18 months in the STAMPEDE trial [24]. Much smaller benefits have been often judged sufficient to change clinical practice in metastatic prostate cancer, as well as in other settings. However, we recognize that a careful selection of patients to be treated with up-front docetaxel is essential for a favorable benefit / risk ratio. Subgroup data of the CHAARTED trial had suggested that the benefit associated with concomitant administration of docetaxel with ADT, at least in early analysis, was more pronounced in patients with "high-volume" disease than in patients with "low-volume" disease [28, 23]. Definition of "high-volume" disease follows previous robust data showing that, in patients with hormone-sensitive disease, the presence of extensive disease (visceral metastases or appendicular skeletal involvement) is related to a worse prognosis [35-37]. In both the CHAARTED - E3805 (based on a prospective definition) and the GETUG-AFU 15 (based on a retrospective assessment), "high-volume" disease was defined as the presence of visceral metastases or the presence of at least 4 bone lesions, with 1 or more lesions in any bone beyond the spine / pelvis. However, based on subgroup data available for those 2 trials $[23,27]$, we performed an exploratory 
analysis of treatment efficacy according to disease volume: although the statistical test for interaction is characterized by a limited statistical power, we did not demonstrate a significant interaction between disease volume and treatment efficacy. Importantly, this absence of significant interaction does not allow to state that the addition of docetaxel to $A D T$ is not effective in patients with low-volume metastatic disease. A longer follow-up with a higher number of events in these latter patients, together with the availability of this subgroup analysis also in the STAMPEDE trial, could increase the statistical power of the analysis. With the currently available evidence, however, no definitive statement can be made about the interaction between docetaxel efficacy and disease volume.

With the exception of a subgroup of patients eligible for the STAMPEDE trial, the majority of patients included in the 3 trials had metastatic disease. Other trials have tested the efficacy of the addition of docetaxel to androgen deprivation therapy in patients with high-risk, localized prostate cancer $[25,26]$. However, the definition of the role of docetaxel in patients with high-risk, localized prostate cancer is beyond the scope of this metaanalysis.

Of course, particular attention should be given to toxicity associated with combination treatment. In the experimental arm of GETUG-AFU 15 study, four treatmentrelated deaths were reported (one due to febrile neutropenia, one neutropenia with infection, one multiorgan failure, and one pulmonary embolism), compared to no treatment-related deaths with ADT alone [22]. In CHAARTED - E3805 trial, only one treatment-related death (sudden death) occurred in combination arm [23]. Although these numbers, overall considered, are quite reassuring, it is well known that patients enrolled in clinical trials are selected compared to all patients treated in daily clinical practice, in terms of age, performance status, comorbidities. For instance, patients older than 70 years are a relevant proportion of those treated in clinical practice, but were quite under-represented in 
371 the 3 trials. In the CHAARTED trial, subgroup analysis according to age supports

372 docetaxel efficacy also in elderly patients, but they represented only $23 \%$ of total study

373 population [23]. Although a potential explanation is that the age of metastatic presentation

374 of patients eligible for these 3 trials could be younger than the whole population of patients

375 with new diagnosis of earlier stage prostate cancer, we believe that the main reason for

376 the under-representation of elderly patients in the trials included in this meta-analysis is

377 the selection bias, because patients had to be fit enough to receive chemotherapy with

378 docetaxel [38]. In any case, chemotherapy toxicity is often worse in the "real world"

379 population, compared with the toxicity reported in clinical trials. Therefore, clinicians must

380 properly take into account some relevant clinical factors (performance status, concomitant

381 diseases) before considering the addition of docetaxel to ADT, in order to reduce the risk

382 of severe toxicity, that could negatively affect quality of life and, in worst cases, survival. In conclusion, our meta-analysis clearly shows a significant impact on overall

384 survival with the concomitant administration of docetaxel and androgen-deprivation

385 treatment in patients with metastatic, hormone-sensitive prostate cancer patients.

386 Considering the absence of heterogeneity among the available trials, and the balance 387 between magnitude of efficacy and risk of toxicity, combination of chemotherapy and 388 hormonal treatment should be reasonably offered to patients with metastatic disease, if 389 judged eligible for chemotherapy. Higher statistical power would be needed to better 390 understand the interaction, if any, between the efficacy of docetaxel and the volume of 391 disease. 
Figure legends.

Figure 1. Forest plots of hazard ratios for overall survival from three randomized trials of docetaxel added to androgen-deprivation therapy (ADT), compared with ADT alone, in patients with advanced, hormone-sensitive prostate cancer. Pooled HRs were computed using random-effect models. The bars indicate $95 \%$ confidence intervals $(\mathrm{Cl})$. Panel $\mathrm{A}$ (only metastatic patients) and panel B (all randomized patients) consider only comparisons between docetaxel + ADT vs. ADT alone. Panel C (only metastatic patients) and panel D (all randomized patients) show a sensitivity analysis considering also the comparison of docetaxel + zoledronic acid + ADT vs. ADT alone in the STAMPEDE trial.

Figure 2. Forest plots of hazard ratios for overall survival (subgroup analysis according to disease volume: patients with "high-volume" disease and patients with "low-volume" disease) in two randomized trials of docetaxel added to androgen-deprivation therapy (ADT), compared with ADT alone, in patients with metastatic, hormone-sensitive prostate cancer. Pooled HRs were computed using random-effect models. The bars indicate 95\% confidence intervals (Cl). Definition of "high-volume" disease and "low-volume" disease is detailed in the text.

Figure 3. Forest plots of hazard ratios for biochemical progression-free survival from three randomized trials of docetaxel added to androgen-deprivation therapy (ADT), compared 414 with ADT alone, in patients with advanced, hormone-sensitive prostate cancer. Pooled HRs were computed using random-effect models. The bars indicate $95 \%$ confidence intervals ( $\mathrm{Cl}$ ). Panel A (only metastatic patients) and panel B (all randomized patients) 417 consider only comparisons between docetaxel + ADT vs. ADT alone. Panel C (only 
418 metastatic patients) and panel D (all randomized patients) show a sensitivity analysis

419 considering also the comparison of docetaxel + zoledronic acid + ADT vs. ADT alone in 420 the STAMPEDE trial.

421

422 Supplemental Figure 1. Selection process of randomized trials eligible for inclusion in 423 the meta-analysis. 


\section{References.}

1. Torre LA, Bray F, Siegel RL, et al. Global Cancer Statistics, 2012. Ca Cancer J Clin 2015;65:87-108.

2. Freedland SJ, Humphreys EB, Mangold LA, et al. Death in patients with recurrent prostate cancer after radical prostatectomy: prostate-specific antigen doubling time subgroups and their associated contributions to all-cause mortality. J Clin Oncol $2007 ; 25: 1765-71$.

3. Siegel R, Miller KD, Jemal A. Cancer statistics, 2015. CA: Cancer J Clin. 2015;65:529.

4. Huggins C, Stevens RE, Hodges CV. Studies on prostatic cancer: II. The effects of castration on advanced carcinoma of the prostate gland. Arch Surg 1941;43:209_ 223.

5. Hellerstedt BA, Pienta KJ. The current state of hormonal therapy for prostate cancer, CA Cancer J Clin 2002;52:154-179.

6. Tannock IF, de Wit R, Berry WR, et al. Docetaxel plus prednisone or mitoxantrone plus prednisone for advanced prostate cancer. N Engl J Med 2004;351:1502-1512.

8. de Bono J, Logothetis C., Molina A, et al. Abiraterone and increased survival in metastatic prostate cancer. N Engl J Med 2011;364:1995-2005.

9. Scher HI, Fizazi K, Saad F, et al. Increased survival with enzalutamide in prostate cancer after chemotherapy. N Engl J Med 2012;367:1187-1197.

10. Kantoff PW, Higano CS, Shore ND, et al. Sipuleucel T immunotherapy for castrateresistant prostate cancer. N Engl J Med 2010;363:411-422. 
11. de Bono JS, Oudard S, Ozguroglu M, et al. Prednisone plus cabazitaxel or mitoxantrone for metastatic castration-resistant prostate cancer progressing after docetaxel treatment: a randomised open-label trial. Lancet 2010;376:1147-1154.

12. Parker C, Nilsson S, Heinrich D, et al. Alpha emitter radium-223 and survival in metastatic prostate cancer. N Engl J Med 2013;369:213-223.

13. Scher HI, Sawyers CK. Biology of progressive, castration-resistant prostate cancer: directed therapies targeting the androgen-receptor signaling axis. J Clin Oncol 2005;23:8253-8261.

14. Nelson PS. Molecular states underlying androgen receptor activation: a framework for therapeutics targeting androgen signalling in prostate cancer. Journal of Clinical Oncology 2012;30:644-646.

15. Mostaghel EA, Marck BT, Plymate SR, et al. Resistance to CYP17A1 inhibition with abiraterone in castration-resistant prostate cancer: induction of steroidogenesis and androgen receptor splice variants. Clin Cancer Res 2011;17:5913-5925.

16. Fenton MA, Shuster TD, Feting Am, et al. Functional characterization of mutant androgen receptors from androgen-independent prostate cancer. Clin Cancer Res $1997 ; 3: 1383-1388$.

17. Antonarakis ES, Lu C, Wang $\mathrm{H}$, et al. AR-V7 and resistance to enzalutamide and abiraterone in prostate cancer. N Engl J Med 2014;371:1028-1038.

18. Stanbrough M, Bubley GJ, Ross K, et al. Increased expression of genes converting adrenal androgens to testosterone in androgen-independent prostate cancer. Cancer Res 2006;66:2815-2825.

19. Ahmed M, Li LC. Adaptation and clonal selection models of castration-resistant prostate cancer: current perspective. Int J Urol 2013;20:362-371. 
20. Millikan RE, Wen S, Pagliaro LC, et al. Phase III trial of androgen ablation with or without three cycles of systemic chemotherapy for advanced prostate cancer. J Clin Oncol 2008; 26: 5936-5942.

21.Wang J, Halford S, Rigg A, Roylance R, Lynch M, Waxman J. Adjuvant mitoxantrone chemotherapy in advanced prostate cancer. BJU Int 2000; 86: 675680.

22. Gravis G, Fizazi K, Joly F, et al. Androgen-deprivation therapy alone or with docetaxel in non-castrate metastatic prostate cancer (GETUG-AFU 15): a randomised, open-label, phase 3 trial. Lancet Oncol 2013;14:149-158.

23. Sweeney CJ, Chen YH, Carducci M, Liu G, Jarrard DF, Eisenberger M, Wong YN, Hahn N, Kohli M, Cooney MM, Dreicer R, Vogelzang NJ, Picus J, Shevrin D, Hussain M, Garcia JA, DiPaola RS. Chemohormonal Therapy in Metastatic Hormone-Sensitive Prostate Cancer. N Engl J Med. 2015 Aug 5. [Epub ahead of print]

24.James ND, Sydes MR, Mason MD, et al.Docetaxel and/or zoledronic acid for hormone-naïve prostate cancer: First overall survival results from STAMPEDE (NCT00268476). J Clin Oncol 33, 2015 (suppl; abstr 5001)

25. Fizazi K, Faivre L, Lesaunier F, et al. Androgen deprivation therapy plus docetaxel and estramustine versus androgen deprivation therapy alone for high-risk localised prostate cancer (GETUG 12): a phase 3 randomised controlled trial. Lancet Oncol 2015;16:787-94.

26. Sandler HM, Hu C, Rosenthal SA, et al. A phase III protocol of androgen suppression (AS) and 3DCRT/IMRT versus AS and 3DCRT/IMRT followed by chemotherapy (CT) with docetaxel and prednisone for localized, high-risk prostate cancer (RTOG 0521). J Clin Oncol 33, 2015 (suppl; abstr LBA5002) 
27. Gravis G, Boher J-M, Joly F, et al. Androgen deprivation therapy (ADT) plus docetaxel (D) versus ADT alone for hormone-naïve metastatic prostate cancer (PCa): Long-term analysis of the GETUG-AFU 15 phase III trial. J Clin Oncol 33, 2015 (suppl 7; abstr 140)

28. Sweeney C, Chen YH, Carducci MA, et al. Impact on overall survival (OS) with chemohormonal therapy versus hormonal therapy for hormone-sensitive newly metastatic prostate cancer (mPrCa): An ECOG-led phase III randomized trial. J Clin Oncol 32:5s, 2014 (suppl; abstr LBA2)

29. Piedbois P, Buyse M. Meta-analyses based on abstracted data: a step in the right direction, but only a first step. J Clin Oncol 2004; 22:3839-3841.

30.Prins GS, Birch L, Greene GL. Androgen receptor localization in different cell types of the adult rat prostate. Endocrinology 1991;129:3187-3199.

31. Karantanos T, Corn PG, Thompson TC. Prostate cancer progression after androgen deprivation therapy: mechanisms of castrate resistance and novel therapeutic approaches. Oncogene 2013;32(49):5501-5511.

32.Darshan MS, Loftus MS, Thadani-Mulero M, et al. Taxane-induced blockade to nuclear accumulation of the androgen receptor predicts clinical responses in metastatic prostate cancer. Cancer Res 2011;71:6019-6029.

33. Thadani-Mulero M, Nanus DM, Giannakakou P. Androgen receptor on the move: boarding the microtubule expressway to the nucleus. Cancer Res 2012;72:46114615.

34.Pienta KJ. Preclinical mechanisms of action of docetaxel and docetaxel combinations in prostate cancer. Semin Oncol 2001;28(4 Suppl 15):3-7.

35. Eisenberger MA, Blumenstein BA, Crawford ED, et al. Bilateral orchiectomy with or without flutamide for metastatic prostate cancer. N Engl J Med 1998;339:10361042. 
36. Crawford ED, Eisenberger MA, McLeod DG, et al. A controlled trial of leuprolide with and without flutamide in prostatic carcinoma. N Engl J Med 1989;321:419-424.

37. Hussain M, Tangen CM, Berry DL, et al. Intermittent versus continuous androgen deprivation in prostate cancer. N Engl J Med 2013;368:1314-1325.

38. James ND, Spears MR, Clarke NW, et al. Survival with Newly Diagnosed Metastatic Prostate Cancer in the "Docetaxel Era": Data from 917 Patients in the Control Arm of the STAMPEDE Trial (MRC PR08, CRUK/06/019). Eur Urol 2015; 67:1028-1038. 


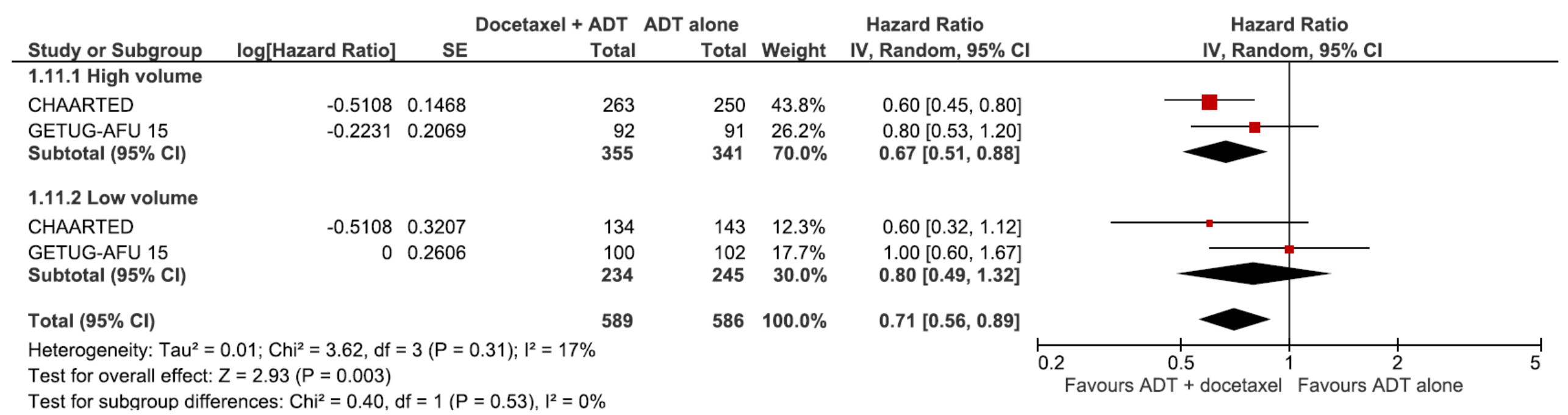




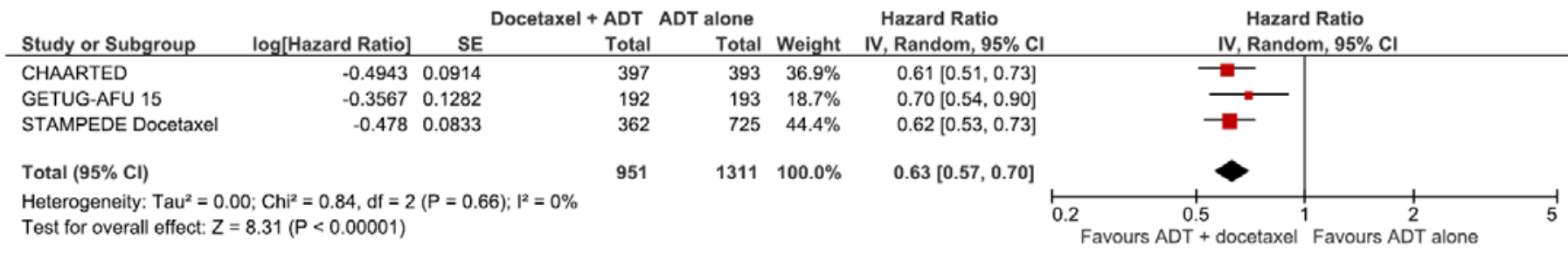

B

Outcome: progression-free survival

(all randomized patients)

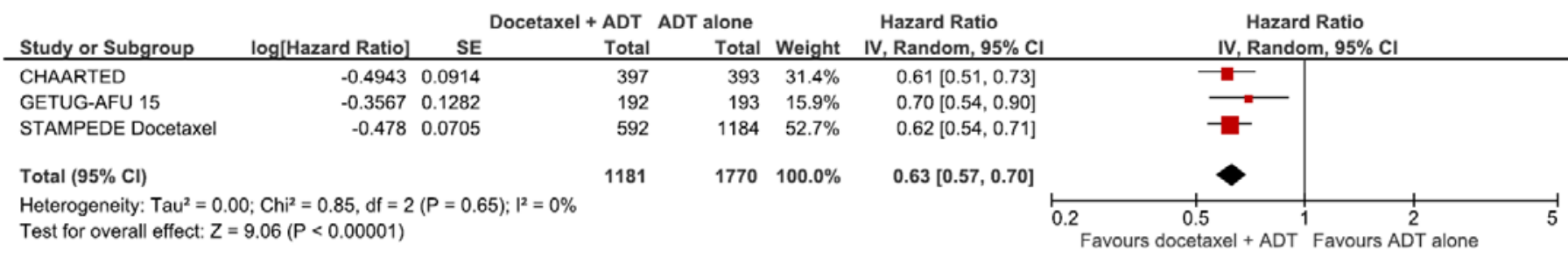

C

Outcome: progression-free survival

(only metastatic patients)

Sensitivity analysis including the docetaxel + zoledronic acid arm

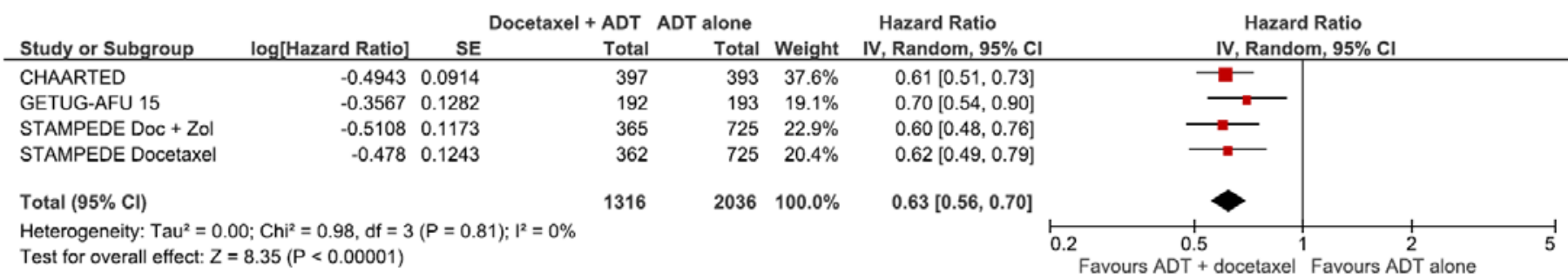

D

Outcome: progression-free survival

(all randomized patients)

Sensitivity analysis including the docetaxel + zoledronic acid arm

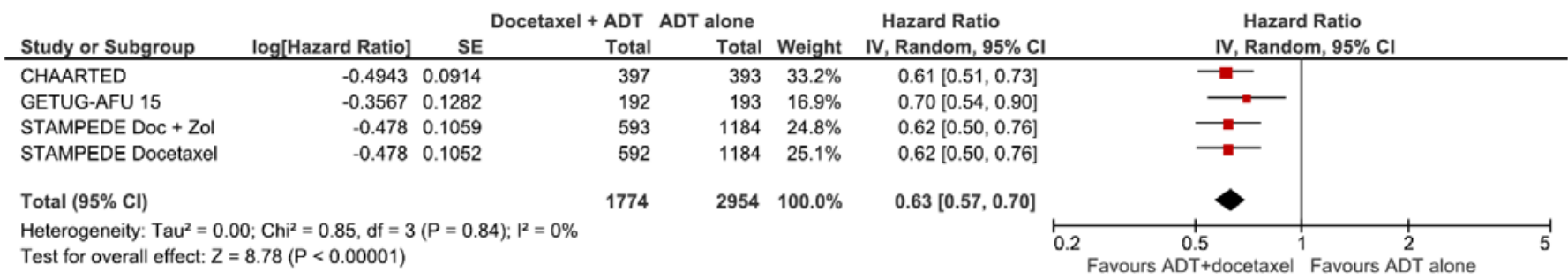


Table 1. Characteristics of the 3 trials included in the meta-analysis

\section{GETUG-AFU 15 CHAARTED - E3805}

$[22,27]$

[23]
STAMPEDE

[24]

\section{Main inclusion criteria}

Age

Older than 18.

Both younger than 70 Not specified.

No upper limit

and older than 70

declared in the

were eligible

methods.

(stratification criteria).

Performance status Karnofsky $>=70$

ECOG 0-2

WHO 0-2

(2 only if due to

prostate cancer)

(0-1 vs 2 stratification

criteria)

Stage

Metastatic prostate

Metastatic prostate

Prostate cancer if

cancer

cancer

metastatic, node-positive

(High-volume vs low-

(High-volume vs low-

or >=2 among:

volume assessed

volume stratification

retrospectively)

criteria)*

- $\quad$ Stage T3/T4

- $P S A>=40 n g / m l$

- Gleason 8-10

Previous treatment

Previous

No prior docetaxel

Prior chemotherapy was

chemotherapy for

was allowed.

not allowed.

metastatic disease

Adjuvant ADT was

Long-term anti-androgen

was not allowed.

allowed, but $<24$

therapy was not allowed.

In the neoadjuvant

months (<=12 months

and adjuvant settings

vs $>12$ months

Short periods of prior

or in the context of

stratification criteria)

anti-androgens to cover

isolated PSA

and interval between

increase, previous

end of adjuvant

chemotherapy or

treatment and

$A D T$, or both, were

progression $>12$

allowed, with the

months.

tumour flare were

allowed.

Adjuvant or neo-adjuvant hormone therapy had to be completed at least 12

condition that the

treatment had been

discontinued at least

12 months before

inclusion in the study.

and duration of

therapy had to be

no longer than 12

months. 
(table continues in next page)

Table 1. (continued)

GETUG+AFU 15 CHAARTED - E3805 STAMPEDE [24]

$[22,26]$

[23]

\begin{tabular}{|c|c|c|c|}
\hline Treatment & & & \\
\hline ADT (both arms) & $\begin{array}{l}\text { Orchiectomy or } \\
\text { LHRH agonists, } \\
\text { alone or combined } \\
\text { with non-steroidal } \\
\text { antiandrogens }\end{array}$ & $\begin{array}{l}\text { Medical or surgical } \\
\text { castration. Use of a } \\
\text { nonsteroidal } \\
\text { antiandrogen at the } \\
\text { time of initiation of } \\
\text { therapy was at the } \\
\text { discretion of the } \\
\text { investigator.. }\end{array}$ & $\begin{array}{l}\text { LHRH analogues or } \\
\text { LHRH antagonists, or } \\
\text { bilateral orchidectomy } \\
\text { according to local } \\
\text { practice }\end{array}$ \\
\hline $\begin{array}{l}\text { Docetaxel } \\
\text { (experimental arm) }\end{array}$ & $\begin{array}{l}\text { Docetaxel }\left(75 \mathrm{mg} / \mathrm{m}^{2}\right. \\
\text { i.v. day } 1 \mathrm{q} 3 \mathrm{w}) \text {; } \\
\text { up to } 9 \text { cycles. } \\
\text { Standard } \\
\text { corticosteroids } \\
\text { premedication, no } \\
\text { daily prednisone. }\end{array}$ & $\begin{array}{l}\text { Docetaxel }\left(75 \mathrm{mg} / \mathrm{m}^{2}\right. \\
\text { i.v. day } 1 \mathrm{q} 3 \mathrm{w}) \text {; } \\
\text { up to } 6 \text { cycles. } \\
\text { Standard } \\
\text { dexamethasone } \\
\text { premedication, no } \\
\text { daily prednisone. }\end{array}$ & $\begin{array}{l}\text { Docetaxel }\left(75 \mathrm{mg} / \mathrm{m}^{2} \mathrm{i} . \mathrm{v} \text {. }\right. \\
\text { day } 1 \mathrm{q} 3 \mathrm{w}) \text {; } \\
\text { up to } 6 \text { cycles. } \\
\text { Standard dexamethasone } \\
\text { premedication, daily } \\
\text { prednisolone } 10 \mathrm{mg} \text {. }\end{array}$ \\
\hline Timing of treatment & $\begin{array}{l}\text { Docetaxel within } 2 \\
\text { months of ADT start. }\end{array}$ & $\begin{array}{l}\text { Docetaxel within } 4 \\
\text { months of ADTstart. }\end{array}$ & $\begin{array}{l}\text { Randomization within } 12 \\
\text { weeks of ADT start. }\end{array}$ \\
\hline $\begin{array}{l}\text { Study design } \\
\text { Primary endpoint }\end{array}$ & Overall survival & Overall survival & Overall survival \\
\hline Hypothesis & $\begin{array}{l}\text { Increase in } 3-y r \text { OS } \\
\text { from } 50 \% \text { to } 65 \%\end{array}$ & $\begin{array}{l}33 \% \text { increase in } \\
\text { median OS } \\
\text { (from } 33 \text { to } 44 \text { months } \\
\text { in high-volume, } \\
\text { from } 67 \text { to } 89 \text { months } \\
\text { in low-volume) }\end{array}$ & $\begin{array}{l}25 \% \text { increase in overall } \\
\text { survival. }\end{array}$ \\
\hline
\end{tabular}

\section{Patients' enrollment and follow-up}

Accrual start

October 2004

Accrual stop

December 2008

Number of patients

ADT alone
October 2005

March 2013
November 2012

393 
ADT + docetaxel

ADT + docetaxel +

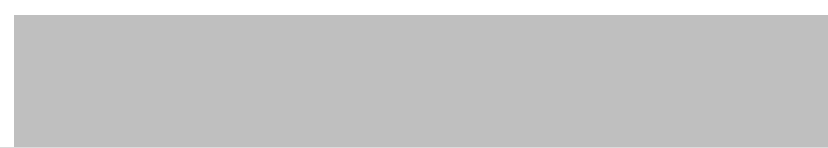

zoledronic acid

Median follow-up
28,9 months
593

n.a.

ADT: androgen-deprivation treatment; ECOG: Eastern Cooperative Oncology Group; WHO: World Health Organization; PSA: prostate-specific antigen; LHRH: luteinizing hormone - releasing hormone; OS: overall survival; n.a.: not available.

*after amendment. In the initial protocol version, only high-volume patients were eligible. 
Table 2. Main characteristics of enrolled patients

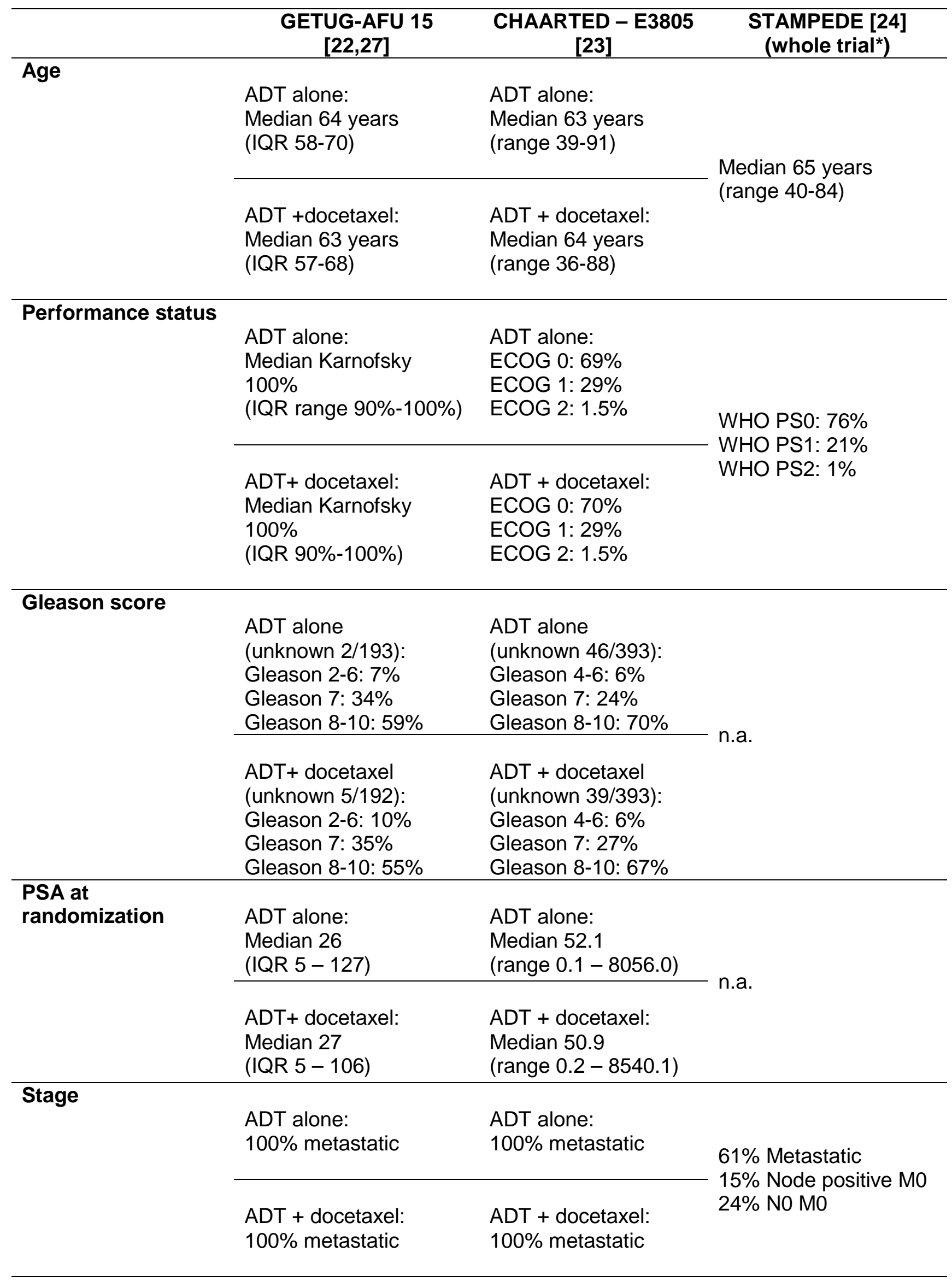




\begin{tabular}{|c|c|c|c|}
\hline \multirow[t]{2}{*}{$\begin{array}{l}\text { Metastatic at } \\
\text { diagnosis }\end{array}$} & $\begin{array}{l}\text { ADT alone: } \\
67 \%\end{array}$ & $\begin{array}{l}\text { ADT alone: } \\
73 \% \text { had not received } \\
\text { prior local therapy }\end{array}$ & \multirow{2}{*}{$\begin{array}{l}94 \% \text { of randomized } \\
\text { patients had not } \\
\text { received previous local } \\
\text { therapy }\end{array}$} \\
\hline & $\begin{array}{l}\text { ADT + docetaxel: } \\
76 \%\end{array}$ & $\begin{array}{l}\text { ADT + docetaxel: } \\
73 \% \text { had not received } \\
\text { prior local therapy }\end{array}$ & \\
\hline
\end{tabular}

\section{Presence of visceral metastases}

$\begin{array}{ll}\text { ADT alone: } & \text { ADT alone: } \\ 11 \% \text { lung } & 17 \% \\ 2 \% \text { liver } & \end{array}$

ADT + docetaxel: $\quad$ ADT + docetaxel:

$11 \%$ lung $\quad 14 \%$

$5 \%$ liver

\begin{tabular}{lll}
\hline $\begin{array}{l}\text { Volume of } \\
\text { metastatic disease }\end{array}$ & ADT alone: \\
& $52 \%$ low-volume & $\begin{array}{l}\text { ADT alone: } \\
36 \% \text { low-volume } \\
48 \% \text { high-volume }\end{array}$ \\
& $64 \%$ high-volume \\
\cline { 2 - 2 } & \\
& ADT + docetaxel: & ADT + docetaxel: \\
& $53 \%$ low-volume & $34 \%$ low-volume \\
$47 \%$ high-volume & $66 \%$ high-volume
\end{tabular}

IQR: interquartile range; ADT: androgen- deprivation treatment; PS: performance status; PSA: prostate specific antigen; MO: absence of distant metastases; NO: absence of nodal metastases; n.a.: not applicable.

*details by arm are not provided 
Table 3. Overall survival data reported in each single trial.

\begin{tabular}{|c|c|c|c|c|}
\hline & \multirow{2}{*}{$\begin{array}{c}\text { GETUG-AFU } 15 \\
{[22,27]}\end{array}$} & \multirow{2}{*}{$\begin{array}{c}\text { CHAARTED - E3805 } \\
\text { [23] }\end{array}$} & \multicolumn{2}{|c|}{ STAMPEDE [24] } \\
\hline & & & All patients & Metastatic patients \\
\hline \multicolumn{5}{|l|}{ Number of patients } \\
\hline ADT alone & 193 & 393 & 1184 & 725 \\
\hline ADT + docetaxel & 192 & 397 & 592 & 362 \\
\hline ADT + docetaxel + zoledronic acid & & & 593 & 365 \\
\hline \multicolumn{5}{|l|}{ Number of events } \\
\hline ADT alone & \multirow{2}{*}{212 (both arms) } & 136 & 405 & 343 \\
\hline ADT + docetaxel & & 101 & 165 & 134 \\
\hline ADT + docetaxel + zoledronic acid & & & 181 & 152 \\
\hline \multicolumn{5}{|l|}{ Median OS } \\
\hline ADT alone & 46.5 months & 44.0 months & 67 months & 43 months \\
\hline ADT + docetaxel & 60.9 months & 57.6 months & 77 months & 65 months \\
\hline ADT + docetaxel + zoledronic acid & & & 72 months & n.a. \\
\hline \multicolumn{5}{|l|}{ Hazard Ratio (95\% confidence interval) } \\
\hline & 0.9 & 0.61 & 0.76 & 0.73 \\
\hline ADT + docetaxel vs. ADT alone & $\begin{array}{c}(0.7-1.2) \\
p=0.4\end{array}$ & $\begin{array}{c}(0.47-0.80) \\
P<0.001\end{array}$ & $\begin{array}{c}(0.63-0.91) \\
p=0.003\end{array}$ & $\begin{array}{c}(0.59-0.89) \\
p=0.002\end{array}$ \\
\hline ADT + docetaxel + zoledronic acid vs. ADT alone & & & $\begin{array}{c}0.81 \\
(0.68-0.97) \\
p=0.02\end{array}$ & $\begin{array}{c}0.78 \\
(0.65-0.95) \\
p=n . a\end{array}$ \\
\hline
\end{tabular}




\section{GETUG-AFU 15 [22] CHAARTED - E3805 [23]}

Time to PSA progression, clinical progression or death. Biochemical progression was defined with the PSA Working Group definition: a previous confirmed PSA decrease of at least $50 \%$ and an increase of at least $50 \%$ above the nadir, with a minimum increase of 5 $\mathrm{ng} / \mathrm{mL}$. For patients without a previous PSA decrease of $50 \%$, progression was defined as a PSA increase of at least $25 \%$ above the nadir and of at least $5 \mathrm{ng} / \mathrm{mL}$.
Time to castration-resistant prostate cancer: time until documented clinical or serologic progression with a testosterone level of less than 50 ng per deciliter (or source documentation of medical castration or surgical castration). Disease progression on imaging was determined according to the Response Evaluation Criteria in Solid Tumors (RECIST), version 1.0.

Serologic progression was defined as an increase in the PSA level of more than $50 \%$ above the nadir reached after the initiation of ADT, with two consecutive increases at least 2 weeks apart. The date of a first recorded increase of more than $50 \%$ above the nadir was deemed the date of progression. If the nadir level was less than $2 \mathrm{ng}$ per milliliter, a minimum increase of more than $2 \mathrm{ng}$ per milliliter was required.
Failure-free survival: First event among PSA failure, local failure, lymph node failure, distant metastases, prostate cancer death.

PSA failure definition: If PSA fall >= 50\%:

- 24 week nadir $+50 \%$ and

- $>4 \mathrm{ng} / \mathrm{ml}$

If PSA fall < 50\%:

- Failure at $\mathrm{t}=0$

PSA: prostate-specific antigen; ADT: androgen deprivation therapy. 